\title{
Extending the bandwidth of electromagnetic cloaks
}

\author{
Huanyang Chen, ${ }^{1,2}$ Zixian Liang, ${ }^{3}$ Peijun Yao, ${ }^{3}$ Xunya Jiang, ${ }^{1,3}$ Hongru Ma, ${ }^{2}$ and C. T. Chan ${ }^{1}$ \\ ${ }^{1}$ Department of Physics, Hong Kong University of Science and Technology, Clear Water Bay, Kowlong, Hong Kong, China \\ ${ }^{2}$ Institute of Theoretical Physics, Shanghai Jiao Tong University, Shanghai 200240, China \\ ${ }^{3}$ Institute of Microsystem and Information Technology, CAS, Shanghai 200050, China
}

(Received 27 November 2007; published 12 December 2007)

\begin{abstract}
Using the idea of a transformation medium, a cloak can be designed to make a domain invisible to one target frequency. We examine the possibility of extending the bandwidth of such a cloak. We find that causality requirements impose severe constraints on the system parameters of the transformation medium, and we show that a specific form of "reduction" can help us to create a cloak that offers a reduced cross section in a finite frequency range. We also give a simple inequality that limits the bandwidth of operation.
\end{abstract}

DOI: 10.1103/PhysRevB.76.241104

PACS number(s): 42.25.Fx, 41.20.Jb, 42.25.Gy

There has always been a keen interest in designing a coating that can make an object invisible, and the idea of using "transformation media" to achieve invisibility has drawn great interest recently. ${ }^{1-6}$ Similar ideas can be used to manipulate electromagnetic waves, such as field concentration, ${ }^{7}$ electromagnetic wormholes, ${ }^{8}$ and field rotation. ${ }^{9}$ It has been noted that an invisibility cloak operates at one single frequency ${ }^{4,5}$ due to causality constraints. ${ }^{10}$ For example, an electromagnetic cloak at microwave frequencies works at $8.5 \mathrm{GHz},{ }^{4}$ while an optical cloak is designed to function at $\lambda=632.8 \mathrm{~nm} .{ }^{5}$ Other mechanisms of cloaking have also been proposed. For example, Alù and Engheta ${ }^{11}$ proposed to shield a dielectric particle by using a plasmonic coating to cancel the lower-order scatterings, and they have shown 12 that their cloaking technique exhibits robustness to frequency and geometric variation. In this paper, we demonstrate how we obtain a reduced scattering cross section (invisibility is the ultimate limit) for a range of frequencies based on the idea of coordinate transformation. ${ }^{1}$ The strategy is to sacrifice the cross section for bandwidth. It is quite clear that we cannot achieve perfect invisibility for an extended range of frequencies, and so we aim at a reduced cross section in a finite frequency range.

We show that the permeability and permittivity determined from a fairly general form of transformation media equations can be realized only in one single frequency, and we suggest a specific "reduced" form of the permeability and permittivity such that the permeability and permittivity will not violate the causality conditions for a finite frequency range. Such a reduced form of transformation media equations gives us a simple way to design a dispersive cloak that operates in a finite frequency range. We show that there are constraints that limit the bandwidth, which is summarized by a simple inequality. We limit ourselves to two-dimensional (2D) systems. In the following, we use the term "dispersive cloak" to mean a cloak that works for a finite bandwidth with the frequency dispersion of the metamaterial taken into consideration.

Using the transformation medium recipe, we generate the ideal 2D invisibility cloak by compressing a cylindrical region, $0 \leq r \leq b$, into a concentric cylindrical shell, $a \leq r \leq b$, by the mapping $r^{\prime}=a+r(b-a) / b$, which results in anisotropic permittivity and permeability in the cloaking shell ${ }^{13}$ of the form

$$
\mu_{r}=\frac{r-a}{r}, \quad \mu_{\theta}=\frac{r}{r-a}, \quad \varepsilon_{z}=\left(\frac{b}{b-a}\right)^{2} \frac{r-a}{r} .
$$

The cloak is equivalent to the free space.

Let us consider a more general transformation that compresses the concentric cylindrical shell region $r_{0} \leq r \leq b$ into another shell $a \leq r \leq b$, with the coordinate transformation $r$ $=b-\left(b-r_{0}\right) /[f(b)-f(a)]\left[f(b)-f\left(r^{\prime}\right)\right]$, where $f(r)$ is a smooth function. The anisotropic permittivity and permeability in the cloaking shell $a \leq r \leq b$ that can give an equivalent distortion of the space are given by

$$
\begin{aligned}
& \mu_{r}=\frac{f(r)-f\left(a^{\prime}\right)}{r f^{\prime}(r)}, \quad \mu_{\theta}=\frac{r f^{\prime}(r)}{f(r)-f\left(a^{\prime}\right)}, \\
& \varepsilon_{z}=\left(\frac{b-r_{0}}{f(b)-f(a)}\right)^{2} \frac{f^{\prime}(r)\left[f(r)-f\left(a^{\prime}\right)\right]}{r},
\end{aligned}
$$

where $f\left(a^{\prime}\right)=\left[b f(a)-r_{0} f(b)\right] /\left(b-r_{0}\right)$ and $f^{\prime}(r)=(d / d r) f(r)$. For the special case of $r_{0}=0$ and $f(r)=r$, Eq. (2) reduces to Eq. (1). If we choose, in particular, $f(r)=r$, then $a^{\prime}=b(a$ $\left.-r_{0}\right) /\left(b-r_{0}\right)$.

The principal refractive indices for Eq. (2) $\operatorname{are}^{10} n_{r}$ $=\sqrt{\varepsilon_{z} \mu_{\theta}}=\left(b-r_{0}\right) /(b-a)$ and $n_{\theta}=\sqrt{\varepsilon_{z} \mu_{r}}=\left[\left(b-r_{0}\right) /(b-a)\right](r$ $\left.-a^{\prime}\right) / r=\left[r\left(b-r_{0}\right)-b\left(a-r_{0}\right)\right] / r(b-a)$.

Such a system has an index ellipsoid of the form ${ }^{14}$

$$
\frac{k_{r}^{2}}{n_{r}^{2}}+\frac{k_{\theta}^{2}}{n_{\theta}^{2}}=\frac{\omega^{2}}{c^{2}}
$$

where $k_{r}$ and $k_{\theta}$ are the components of the propagation vector in polar coordinates, $\vec{k}=k_{r} \hat{r}+k_{\theta} \hat{\theta}(\hat{r}$ and $\hat{\theta}$ are radial and tangential unit vectors for every point in real space inside the cloak). The magnitude of the group velocity is then ${ }^{15}$

$$
v_{g}=\frac{\omega \sqrt{\frac{k_{r}^{2}}{n_{r}^{4}}+\frac{k_{\theta}^{2}}{n_{\theta}^{4}}}}{\frac{k_{r}^{2}}{n_{r}^{2}} \frac{n_{r}+\omega \frac{d n_{r}}{d \omega}}{n_{r}}+\frac{k_{\theta}^{2}}{n_{\theta}^{2}} \frac{n_{\theta}+\omega \frac{d n_{\theta}}{d \omega}}{n_{\theta}} .}
$$

We must make sure that the group velocity $v_{g} \leq c$ is in every direction. There are two necessary conditions such that 
$v_{g} \leq c$. They are $v_{g}=c /\left(n_{\theta}+\omega d n_{\theta} / d \omega\right) \leq c$ when $k_{\theta}$ $=(\omega / c) n_{\theta}\left(k_{r}=0\right)$ and $v_{g}=c /\left(n_{r}+\omega d n_{r} / d \omega\right) \leq c$ when $k_{r}$ $=(\omega / c) n_{r}\left(k_{\theta}=0\right)$. These imply that $n_{r}+\omega d n_{r} / d \omega$ $\geq 1 \Rightarrow \omega d r_{0} / d \omega \leq a-r_{0}$ and $n_{\theta}+\omega d n_{\theta} / d \omega \geq 1 \Rightarrow \omega d r_{0} / d \omega$ $\geq a-r_{0}$. The above two inequalities result in two conflicting conditions for all frequencies except for the trivial solution $a=r_{0}$. One potential nontrivial solution is that $\omega d r_{0} / d \omega=a$ $-r_{0}$. However, on substituting the solution of this equation into Eq. (4), we will find that $v_{g}=c$ for $\hat{r}$ and $\hat{\theta}$, and $v_{g}>c$ otherwise. We can show that the more general form of Eq. (2) encounters the same problem.

The result shows that no analytic function $r_{0}(\omega)$ can satisfy the causality requirement. In other words, the generalized transformation media equations given by Eq. (2) can be realized in only one single frequency, and if a material obeys the transformation media equations in one frequency, the $\varepsilon$ and $\mu$ cannot satisfy the transformation media equations in any other nearby frequency. This also means that, in all likelihood, the scattering cross section will increase rapidly once we move away from the target frequency.

For ease of fabrication, there are several methods of reduction, ${ }^{4,5}$ which lump all the spatial dependence into to just $\mu_{r}$. For instance, we can have

$$
\mu_{r}=\left(\frac{r-a^{\prime}}{r}\right)^{2}, \quad \mu_{\theta}=1, \quad \varepsilon_{z}=\left(\frac{b-r_{0}}{b-a}\right)^{2} .
$$

Such reduction preserves the index ellipsoid, and thus the transformation media cannot be extended to a finite bandwidth even if we allow $r_{0}(\omega)$ to be an arbitrary function of the frequency.

Let us now discuss what motivates us to consider the general form of Eq. (2) in the first place. To achieve an invisible cloak, we would like to set $r_{0}=0$. It is quite obvious that, if we choose to set $r_{0}=0$ for one frequency, say $\omega_{a}$, we cannot set $r_{0}=0$ for another nearby frequency $\omega_{a}+\Delta \omega$, since that would require that we have frequency-independent permittivity and permeability. For the reason that metamaterials are intrinsically dispersive, this is not possible. But suppose that the generalized form of the transformation media [Eq. (2)] can be satisfied for a range of frequencies. We may then attempt to construct a dispersive system such that $r_{0}(\omega)$ is zero at one frequency and $r_{0}(\omega)$ is small (but finite) for a certain frequency range. We hope that by sacrificing the cross section for the bandwidth, we can achieve a finitebandwidth "partial" cloak that has a zero cross section in one frequency and a very small cross section in nearby frequencies. However, the index ellipsoid consideration shows that this is an impossible task. We also know that the usual form of reduction, which preserves the refractive index within the cloak, will not help.

In the following, we will show that a slightly modified form of the transformation media equations yields an index ellipsoid that gives $v_{g} \leq c$ in every direction. Consider the form

$$
\mu_{r}=\frac{r-a^{\prime}}{r}, \quad \mu_{\theta}=\frac{r}{r-a^{\prime}}, \quad \varepsilon_{z}=\left(\frac{b-\bar{r}}{b-a}\right)^{2} \frac{r-a^{\prime}}{r},
$$

with its corresponding reduction

$$
\mu_{r}=\left(\frac{r-a^{\prime}}{r}\right)^{2}, \quad \mu_{\theta}=1, \quad \varepsilon_{z}=\left(\frac{b-\bar{r}}{b-a}\right)^{2},
$$

where $\bar{r}$ is a very slow varying function (treated as a constant) over a certain frequency range. Note that in Eq. (5) $a^{\prime}$ is a function of $r_{0}$, while in Eq. (7) $a^{\prime}$ and $\bar{r}$ are independent.

The principal refractive indexes are then $n_{r}=\sqrt{\varepsilon_{z} \mu_{\theta}}=(b$ $-\bar{r}) /(b-a)$ and $n_{\theta}=\sqrt{\varepsilon_{z} \mu_{r}}=[(b-\bar{r}) /(b-a)]\left(r-a^{\prime}\right) / r$. We see that $n_{r}$ is not a function of the frequency but $n_{\theta}$ still is.

If we introduce an auxiliary angle such that $k_{r}$ $=(\omega / c) n_{r} \cos \tau$ and $k_{\theta}=(\omega / c) n_{\theta} \sin \tau$, the causality condition $\left(v_{g} \leq c\right)$ can be written as

$$
\frac{v_{g}}{c}=\frac{\sqrt{\frac{n_{\theta}^{2}}{n_{r}^{2}} \cos ^{2} \tau+\sin ^{2} \tau}}{n_{\theta} \cos ^{2} \tau+\left(n_{\theta}+\omega \frac{d n_{\theta}}{d \omega}\right) \sin ^{2} \tau} \leq 1 .
$$

We note that this condition is not always satisfied. ${ }^{16}$

However, we will show by explicit construction that the conditions can be satisfied in some cases. We should emphasize that Eq. (7) is not the only way to design a cloak that operates in a finite frequency range, but it is a convenient way of doing so while sticking as close as possible to the original transformation media equations, which are known to offer perfect invisibility at one frequency.

We can extract some important intrinsic physical information by considering the direction $k_{r}=0, n_{\theta}+\omega d n_{\theta} / d \omega$ $\geq 1 \Rightarrow \omega d r_{0} / d \omega \geq[b /(b-\bar{r})] a$. Since $\bar{r}$ is taken to be much smaller than $b$, we have the inequality

$$
\omega \frac{d r_{0}}{d \omega} \geq a \Rightarrow \frac{\Delta \omega}{\omega} \leq \frac{\Delta r_{0}}{a} .
$$

This is a necessary condition. This invisibility condition sets a limit on the bandwidth of the type of reduced transformation media equation as specified by Eq. (7). The physical meaning of this condition is clear. If we hope to be invisible in a larger frequency range with cloaking based on geometric transformation, we must have more tolerance for $r_{0}$ (the cross section is larger).

Based on Eq. (7), we present a dispersive cloak design working in a microwave frequency range with its metamaterial implemented as an array of split ring resonators (SRRs). The inner and outer radii are $a=27.1 \mathrm{~mm}$ and $b=58.9 \mathrm{~mm}$, the same as in an experimental case. ${ }^{4}$ We set $r_{0} \quad(\omega$ $=8.5 \mathrm{GHz})=0 \mathrm{~mm}$ and $r_{0}^{\max }=r_{0}(\omega=8.75 \mathrm{GHz})=4 \mathrm{~mm}$. And we set $\bar{r}=r_{0}^{\mathrm{av}}=\left(0+r_{0}^{\max }\right) / 2=2 \mathrm{~mm}$, which means that $\varepsilon_{z}$ $=[(b-\bar{r}) /(b-a)]^{2}=3.2$. With these parameters, we can obtain from Eq. (7) the designed $\mu_{r}$ at 8.5 and at $8.75 \mathrm{GHz}$. To obtain a particular design, we need a certain metamaterial to realize the designed $\mu_{r}$. The SRR medium is chosen because it is a well-developed and widely used form of metamaterial ${ }^{17,18}$ whose dispersion relation can be written as

$$
\mu=1-\frac{F f^{2}}{f^{2}-f_{0}^{2}+i f \gamma},
$$

where $\gamma=0.01 \mathrm{GHz}$. We note that this form is expected to work only for a limited frequency range, and more complex 
TABLE I. The SSR parameters for each shell.

\begin{tabular}{lcc}
\hline \hline Shell & $F$ & $f_{0}(\mathrm{GHz})$ \\
\hline 1 & 0.777566 & 3.98772 \\
2 & 0.653523 & 4.89096 \\
3 & 0.571633 & 5.35577 \\
4 & 0.512269 & 5.63920 \\
5 & 0.468967 & 5.80921 \\
6 & 0.431116 & 5.95249 \\
7 & 0.403528 & 6.02870 \\
8 & 0.378390 & 6.09923 \\
9 & 0.355596 & 6.16397 \\
10 & 0.337370 & 6.20024 \\
\hline \hline
\end{tabular}

functions would be needed for a very broad range consideration. This is not an issue here since the cloak has limited bandwidth in any case. Following the reported microwave experimental technique, we break the continuous cloaking material into ten shells in the radial direction. ${ }^{4}$ We determine the SSR parameters $F$ and $f_{0}$ for each shell by requiring the real part of Eq. (10) to be equal to the $\mu_{r}$ of Eq. (7) at 8.5 and $8.75 \mathrm{GHz}$. Table I shows the SSR parameters for each shell.

To show the advantage of this dispersive cloak, we also construct a single-frequency cloak as in Ref. 4 . We assume a Lorenzian form of dispersion (other dispersion relations such as the Drude model give similar results) of the singlefrequency cloak material: ${ }^{19}$

$$
\mu=1-\frac{f_{a}^{2}}{f^{2}-f_{0}^{2}+i f \gamma},
$$

where $f_{0}$ and $\gamma$ are the same as in the SRR of our design, but $f_{a}=\sqrt{F} 8.5 \mathrm{GHz}$ to obtain the required $\mu_{r}$ at $8.5 \mathrm{GHz}\left(r_{0}=0\right.$ case) and $\varepsilon_{z}$ is 3.43 .

Figure 1 shows the normalized total scattering cross section [normalized to a bare perfect electrical conductor (PEC) cylinder with radius $a=27.1 \mathrm{~mm}$ ] at different frequencies of our dispersive cloak and the single-frequency cloak. We first calculate the total cross section of a PEC cylinder for differ-

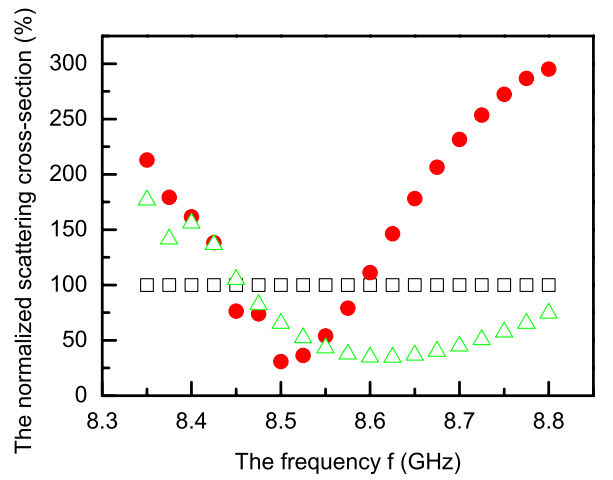

FIG. 1. (Color online) Normalized total scattering cross section as a function of frequencies for the dispersive cloak (triangles), the single-frequency cloak (circles), and the PEC cylinder (squares).
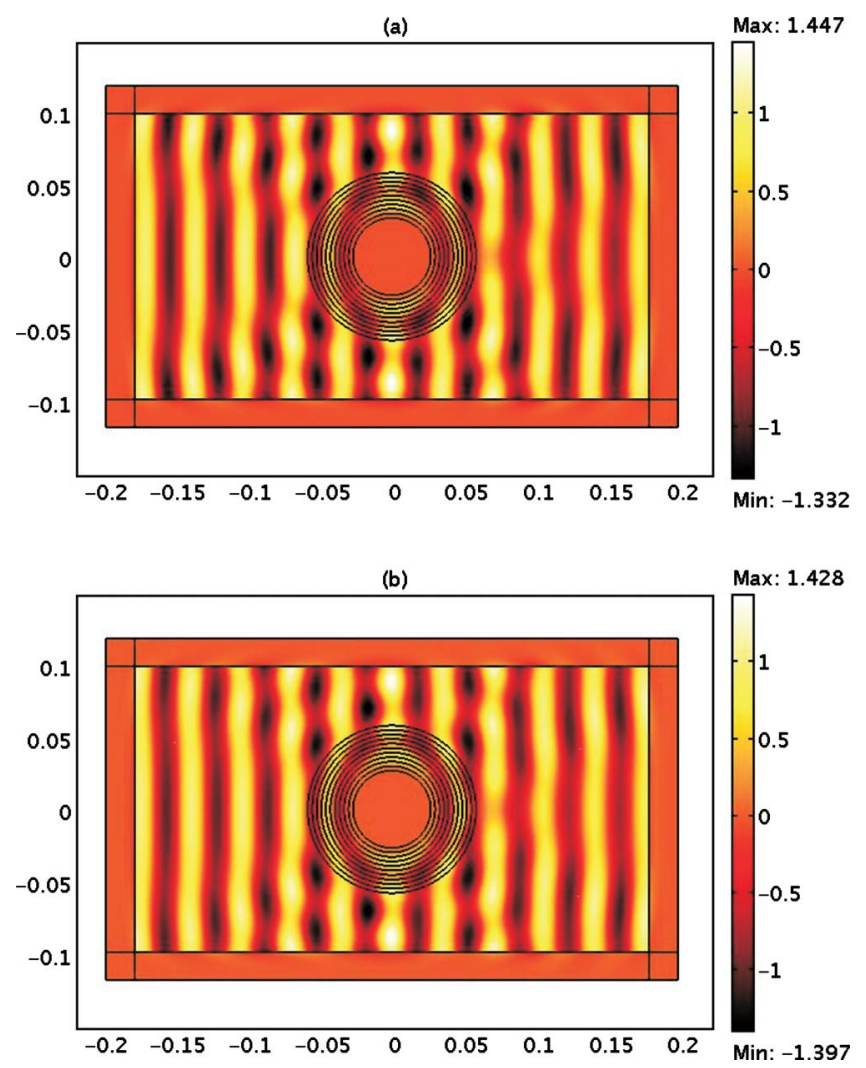

FIG. 2. (Color online) (a) Electric-field distribution in the vicinity of the dispersive cloak at $8.625 \mathrm{GHz}\left(\varepsilon_{z}=3.2\right)$. (b) Same as (a) but for the original cloak at $8.5 \mathrm{GHz}\left(\varepsilon_{z}=3.43\right)$.

ent frequencies. We then calculate the total cross section for both the dispersive cloak and the single-frequency cloak, and normalize them to the PEC case for different frequencies. We find that the dispersive cloak works in a broader frequency range than the original one.

In such reduced cloaks, the cross section is not zero. The minimum cross section is about $30 \%$ of the bare cylinder for both the dispersive cloak and the single-frequency cloak at that range, as shown in Fig. 2, where we compare the scattering pattern of the dispersive cloak (at $8.625 \mathrm{GHz}$, the central frequency of the working bandwidth) and the original cloak at $8.5 \mathrm{GHz}$. The incident TE plane wave is from left to right in the $x$ direction. ${ }^{20}$ The results are calculated using the

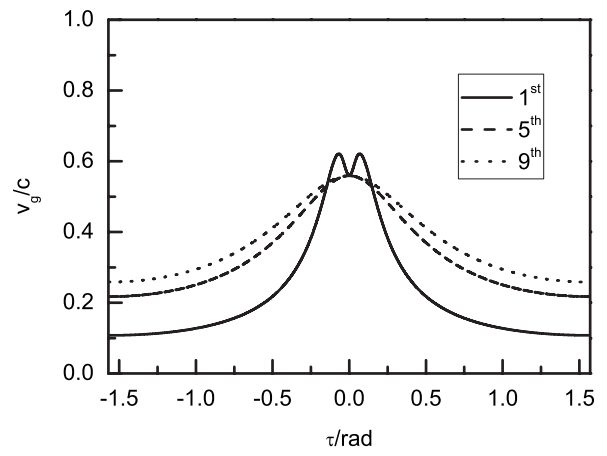

FIG. 3. Dependence of $v_{g} / c$ on the auxiliary angle $\tau$ (see text for details). 
finite-element solver COMSOL MULTIPHYSICS (our own finitedifference time-domain code gives nearly the same results). We note that $\mu_{r}$ at a frequency of $8.625 \mathrm{GHz}$ does not follow Eq. (7) strictly but it is extracted from the SRR model, and the cloaking effect is no worse than for the original cloak at $8.5 \mathrm{GHz}$. The frequency of the minimum cross section for the dispersive cloak can be tuned by the value of $\bar{r}$.

Finally, we emphasize that the parameters chosen here have been tested to ensure that the causality constraint [Eq. (8)] is satisfied. As an example, we calculate $v_{g} / c$ as a function of the parametric angle $\tau$ at the frequency of $8.5 \mathrm{GHz}$ for the inner most shell (the first), the fifth shell, and the ninth shell. We find that $v_{g} / c$ is always smaller than 1 , as shown in Fig. 3.

In conclusion, we have shown that a generalized form of the transformation media equations can be realized at one single frequency, but it cannot be made to be compatible with causality over an extended range of frequencies, no matter how small that range is. However, by a simple adaptation, the reduced form transformation media equations can be made to be compatible with the causality requirements, which then leads to a simple way of designing a reducedcross-section cloak for a finite range of frequencies. We found that the reduced media equations obey a simple invisibility condition, which limits the bandwidth. An explicit example is constructed to illustrate the idea.

This work was supported by a Hong Kong Central Allocation Grant No. HKUST3/06C. The computation resources were supported by the Shun Hing Education and Charity Fund. X. J. acknowledges support from NKBRSF Grant No. 2006CB921701-6.
${ }^{1}$ J. B. Pendry, D. Schurig, and D. R. Smith, Science 312, 1780 (2006).

${ }^{2}$ U. Leonhardt, Science 312, 1777 (2006); Nat. Photonics 1, 207 (2007).

${ }^{3}$ A. Greenleaf, M. Lassas, and G. Uhlmann, Math. Res. Lett. 10, 685 (2003); A. Greenleaf, M. Lassas, and G. Uhlmann, Physiol. Meas 24, 413 (2003).

${ }^{4}$ D. Schurig, J. J. Mock, B. J. Justice, S. A. Cummer, J. B. Pendry, A. F. Starr, and D. R. Smith, Science 314, 977 (2006).

${ }^{5}$ W. Cai U. K. Chettiar, A. V. Kildishev, and V. M. Shalaev, Nat. Photonics 1, 224 (2007).

${ }^{6}$ H. Chen and C. T. Chan, Appl. Phys. Lett. 91, 183518 (2007).

${ }^{7}$ M. Rahm, D. Schurig, D. A. Roberts, S. A. Cummer, D. R. Smith and J. B. Pendry, arXiv:0706.2452 (unpublished).

${ }^{8}$ A. Greenleaf, Y. Kurylev, M. Lassas, and G. Uhlmann, Phys. Rev. Lett. 99, 183901 (2007).

${ }^{9}$ H. Chen and C. T. Chan, Appl. Phys. Lett. 90, 241105 (2007).

${ }^{10}$ B. Wood and J. B. Pendry, J. Phys.: Condens. Matter 19, 076208 (2007).

${ }^{11}$ A. Alù and N. Engheta, Phys. Rev. E 72, 016623 (2005).

${ }^{12}$ A. Alù and N. Engheta, Opt. Express 15, 3318 (2006).
${ }^{13}$ S. A. Cummer, B.-I. Popa, D. Schurig, D. R. Smith, and J. B. Pendry, Phys. Rev. E 74, 036621 (2006).

${ }^{14}$ D. Schurig, J. B. Pendry, and D. R. Smith, Opt. Express 14, 9794 (2006).

${ }^{15}$ L. D. Landau and E. M. Lifshitz, Electrodynamics of Continuous Media (Pergamon Press, Oxford, 1975), Chap. 11, pp. 315-321.

${ }^{16} \mathrm{We}$ avoid considering frequency ranges in which the material have strong anomalous dispersion (e.g., near-resonant absorption) since considerable absorption defeats the purpose of invisibility cloaking. In those frequency ranges, the group velocity is not directly related to causality.

${ }^{17}$ J. B. Pendry, A. J. Holden, D. J. Robbins, and W. J. Stewart, IEEE Trans. Microwave Theory Tech. 47, 2075 (1999).

${ }^{18}$ D. R. Smith, Willie J. Padilla, D. C. Vier, S. C. Nemat-Nasser, and S. Schultz, Phys. Rev. Lett. 84, 4184 (2000).

${ }^{19}$ T. Koschny, M. Kafesaki, E. N. Economou, and C. M. Soukoulis, Phys. Rev. Lett. 93, 107402 (2004).

${ }^{20}$ Note that the mapping keeps the PEC boundary from $r=r_{0}$ to $r$ $=a$ so that the PEC is at $r=a$. As the imaginary parts of $\mu_{r}$ are smaller than 0.002 due to the small $\gamma$, we omit the absorption here. 